\title{
Decay of magnetohydrodynamic turbulence from power-law initial conditions
}

\author{
Chirag Kalelkar* and Rahul Pandit ${ }^{\dagger}$ \\ Centre for Condensed Matter Theory, Department of Physics, Indian Institute of Science, Bangalore 560012, India
}

\begin{abstract}
We derive relations for the decay of the kinetic and magnetic energies and the growth of the Taylor and integral scales in unforced, incompressible, homogeneous, and isotropic three-dimensional magnetohydrodynamic (3DMHD) turbulence with power-law initial energy spectra. We also derive bounds for the decay of the cross and magnetic helicities. We then present results from systematic numerical studies of such decay both within the context of a MHD shell model and direct numerical simulations of 3DMHD. We show explicitly that our results about the power-law decay of the energies hold for times $t<t_{*}$, where $t_{*}$ is the time at which the integral scales become comparable to the system size. For $t<t_{*}$, our numerical results are consistent with those predicted by the principle of "permanence of large eddies."
\end{abstract}

\section{INTRODUCTION}

The decay of homogeneous and isotropic fluid turbulence has been the subject of extensive theoretical $[1,2]$ and experimental [3-5] studies. These include wind-tunnel experiments downstream of a grid [3] and flow behind a towed grid in a stationary channel filled with Helium II [4]. However, unambiguous statements can still not be made about the dependence of the decay of fluid turbulence on the initial conditions. In experiments, these can be changed to some extent by changing the grid geometry and choice of virtual origin [5] but caution is advised in interpreting the results since our ability to choose initial conditions precisely is severely restricted. In an earlier study [6], the energy-decay exponents were predicted to be initial-condition dependent, but the precise nature of this dependence was not elucidated clearly. Direct numerical simulations (DNS) can control initial conditions, but, to the best of our knowledge, have investigated a very limited class of initial conditions. A recent exception [7] is a study of the decay of fluid turbulence for the set of initial conditions with a power-law initial energy spectrum $E^{0}(k) \sim k^{q}$, where the superscript 0 denotes the choice of virtual origin of time $t=t_{0}, k$ is the magnitude of the wave vector, and the exponent $q$ distinguishes different initial conditions in this set.

Results, both experimental and numerical, for the decay of magnetohydrodynamic (MHD) turbulence are even more scarce than their fluid-turbulence analogs. Different powerlaw decays have been suggested in DNS [9] studies, but the sensitivity of these decays to the initial conditions or evolution of the relevant length scale has not been investigated in any detail.

In this paper, we initiate such an investigation and obtain the following interesting results: We show first how to generalize the results of Refs. [7,8] and derive, for unforced, incompressible, homogeneous, and isotropic [10] three-

\footnotetext{
*Electronic address: kalelkar@physics.iisc.ernet.in

${ }^{\dagger}$ Also at the Jawaharlal Nehru Centre for Advanced Scientific Research, Bangalore, India.

Electronic address: rahul@physics.iisc.ernet.in
}

dimensional MHD (3DMHD) turbulence, expressions for the decay of the kinetic and magnetic energies and the growth of the Taylor and integral scales, when we start with power-law initial conditions $E_{\mathbf{a}}^{0}(k) \sim k^{q}(q>-1)$, where the subscript a is $\mathbf{v}$ for the kinetic energy and $\mathbf{b}$ for the magnetic energy. Such initial conditions are of interest in the astrophysical context of the decay of power-law "primordial" energy spectra [8]. We also derive bounds for the decay of the cross and magnetic helicity. We then show by systematic numerical studies of a shell model for MHD turbulence [11,12] and an $80^{3}$ pseudospectral DNS of the 3DMHD equations that, given power-law initial conditions, the kinetic and magnetic energies and the Taylor and integral scales follow the decay expressions mentioned above within a regime governed by the temporal evolution of the integral scales.

The unforced MHD equations are

$$
\begin{gathered}
\frac{\partial \mathbf{v}}{\partial t}+(\mathbf{v} \cdot \nabla) \mathbf{v}=-\frac{\nabla p^{*}}{\rho}+\frac{(\mathbf{b} \cdot \boldsymbol{\nabla}) \mathbf{b}}{4 \pi \rho}+\nu \nabla^{2} \mathbf{v}, \\
\frac{\partial \mathbf{b}}{\partial t}=\nabla \times(\mathbf{v} \times \mathbf{b})+\eta \nabla^{2} \mathbf{b},
\end{gathered}
$$

where $\nu$ is the kinematic viscosity, $\eta$ is the magnetic viscosity, $p^{*}=p+b^{2} / 8 \pi$ is the effective pressure, and $p$ is the pressure. We enforce the incompressibility condition $\boldsymbol{\nabla} \cdot \mathbf{v}=\mathbf{0}$ and eliminate $p^{*}$ in the usual manner. The invariants of inviscid, unforced 3DMHD are the total energy $\left[E_{T}=E_{\mathbf{v}}+E_{\mathbf{b}}\right.$ $\left.\equiv \frac{1}{2} \int\left(v^{2}+b^{2}\right) d V\right]$, the cross helicity $\left(H_{C} \equiv \int \mathbf{v} \cdot \mathbf{b} d V\right)$, and the magnetic helicity $\left(H_{M} \equiv \int \mathbf{A} \cdot \mathbf{b} d V\right.$, where $\mathbf{A}$ is the vector potential). We restrict ourselves to the case with $\left|H_{M}^{0}\right|$ close to zero and exclude any helical contributions [9] to the decay process due to the inverse cascade of $H_{M}$. Most of our results are obtained for initial equipartition of energy, namely, $E_{\mathbf{v}}^{0}$ $=E_{\mathbf{b}}^{0}$, but we also present a few results for $E_{\mathbf{v}}^{0} \neq E_{\mathbf{b}}^{0}$.

\section{THEORETICAL RESULTS}

We observe that Eqs. (1) are invariant under the rescaling

$$
\mathbf{x} \rightarrow l \mathbf{x}, \quad t \rightarrow l^{1-h} t, \quad \mathbf{v} \rightarrow l^{h} \mathbf{v}, \quad \mathbf{b} \rightarrow l^{h} \mathbf{b},
$$




$$
\nu \rightarrow l^{1+h} \nu, \quad \eta \rightarrow l^{1+h} \eta
$$

where $l$ is an arbitrary scale factor and $h<0$ a scaling exponent. The local kinetic- and magnetic-energy densities in three dimensions are given by

$$
\begin{aligned}
\mathcal{E}_{\mathbf{a}}(k, t, \nu, \eta, K, 2 \pi / L)= & \frac{\Omega k^{2}}{(2 \pi)^{3} L^{3}} \int_{2 \pi / K}^{L} d^{3} x d^{3} y e^{i \mathbf{k} \cdot(\mathbf{x}-\mathbf{y})} \\
& \times\langle\mathbf{a}(\mathbf{x}, t) \cdot \mathbf{a}(\mathbf{y}, t)\rangle
\end{aligned}
$$

where $L$ and $2 \pi / K$ are the large- and small-distance cutoffs, the subscript $\mathbf{a}$ is $\mathbf{v}$ for the kinetic-energy density and $\mathbf{b}$ for the magnetic-energy density, and $\Omega$ is the solid angle. Angular brackets denote an average over the initial conditions being considered (see below). Thus the kinetic and magnetic energies in this range of length scales are

$$
E_{\mathbf{a}}(t, \nu, \eta, 2 \pi / K, L)=\int_{2 \pi / L}^{K} d k \mathcal{E}_{\mathbf{a}}(k, t, \nu, \eta, K, 2 \pi / L) .
$$

By combining Eqs. (2) and (3) we get

$$
\begin{gathered}
\mathcal{E}_{\mathbf{a}}\left(k / l, l^{1-h} t, l^{1+h} \nu, l^{1+h} \eta, K / l, 2 \pi / L l\right) \\
=l^{1+2 h} \mathcal{E}_{\mathbf{a}}(k, t, \nu, \eta, K, 2 \pi / L) .
\end{gathered}
$$

Henceforth the dependence of $\mathcal{E}_{\mathrm{a}}$ on $K$ and $2 \pi / L$ will be suppressed for notational convenience. It is useful to define the functions

$$
\Phi_{\mathbf{a}}(k, t, \nu, \eta) \equiv k^{1+2 h} \mathcal{E}_{\mathbf{a}}(k, t, \nu, \eta)
$$

in terms of which Eq. (5) becomes

$$
\Phi_{\mathbf{a}}\left(k / l, l^{1-h} t, l^{1+h} \nu, l^{1+h} \eta\right)=\Phi_{\mathbf{a}}(k, t, \nu, \eta)
$$

when differentiated with respect to $l$, this yields, in the limit $l \rightarrow 1$,

$$
-\frac{\partial \Phi_{\mathbf{a}}}{\partial \ln k}+(1-h) \frac{\partial \Phi_{\mathbf{a}}}{\partial \ln t}+(1+h) \frac{\partial \Phi_{\mathbf{a}}}{\partial \ln \nu}+(1+h) \frac{\partial \Phi_{\mathbf{a}}}{\partial \ln \eta}=0
$$

The general solution of the above equation is

$$
\begin{aligned}
\Phi_{\mathbf{a}}(k, t, \nu, \eta)= & F_{\mathbf{a}}((1-h) \ln k+\ln t,(1+h) \ln t \\
& +(-1+h) \ln \nu,(1+h) \ln t+(-1+h) \ln \eta) .
\end{aligned}
$$

This solution corresponds to

$$
\mathcal{E}_{\mathbf{a}}(k, t, \nu, \eta)=k^{q} \Phi_{\mathbf{a}}\left(k^{(3+q) / 2} t, t^{(1-q) / 2} \nu^{-(3+q) / 2}, t^{(1-q) / 2} \eta^{-(3+q) / 2}\right),
$$

where $q=-1-2 h(q>-1)[13]$.

For $q=1$, Eqs. (4) and (10) lead to especially simple forms for the temporal decay of the kinetic and magnetic energies. We are interested in the limits $2 \pi / L \rightarrow 0$ and $K$ $\rightarrow \infty$, of relevance to high-Reynolds-number turbulence, so

$$
E_{\mathbf{a}}(t)=\frac{1}{2 t} \int_{0}^{\infty} d y \Phi_{\mathbf{a}}\left(y, 1 / \nu^{2}, 1 / \eta^{2}\right) .
$$

Thus both $E_{\mathbf{v}}$ and $E_{\mathbf{b}}$ decay as $1 / t$, which generalizes the result of Ref. [7] to the case of decaying MHD turbulence. For all $q \neq 1$, the simple power-law dependence of $E_{\mathbf{a}}(t)$ on $t$ does not follow. The fluid and magnetic Taylor scales, $\quad \lambda_{\mathbf{a}}(t, \nu, \eta, 2 \pi / K, L) \equiv\left[\int_{2 \pi / L}^{K} d k \mathcal{E}_{\mathbf{a}}(k, t, \nu, \eta)\right.$ $\left./ \int_{2 \pi / L}^{K} d k k^{2} \mathcal{E}_{\mathbf{a}}(k, t, \nu, \eta)\right]^{1 / 2}$, also show a simple power-law dependence on $t$ only for $q=1$. In the limits $2 \pi / L \rightarrow 0, K$ $\rightarrow \infty$,

$$
\lambda_{\mathbf{a}}(t)=t^{1 / 2}\left[\frac{\int_{0}^{\infty} d y \Phi_{\mathbf{a}}\left(y, 1 / \nu^{2}, 1 / \eta^{2}\right)}{\int_{0}^{\infty} d y \Phi_{\mathbf{a}}\left(y, 1 / \nu^{2}, 1 / \eta^{2}\right) y}\right]^{1 / 2} .
$$

Similarly, the integral scales $L_{\mathbf{a}}(t, \nu, \eta, 2 \pi / K, L)$ $\equiv\left[\int_{2 \pi / L}^{K} d k \mathcal{E}_{\mathbf{a}}(k, t, \nu, \eta) / k\right] / \int_{2 \pi / L}^{K} d k \mathcal{E}_{\mathbf{a}}(k, t, \nu, \eta)$ (where the subscript $\mathbf{a}$ is $\mathbf{v}$ for the fluid integral scale and $\mathbf{b}$ for the magnetic integral scale), also grow as a power of $t$ with exponent equal to 0.5 for $q=1$ in the limits $2 \pi / L \rightarrow 0, K$ $\rightarrow \infty$.

A consequence of the positive definiteness of the energy spectra is that the helicity spectra satisfy realizability constraints $\left|\mathcal{H}_{C}(k, t, \nu, \eta)\right| \leqslant\left[\mathcal{E}_{\mathbf{v}}(k, t, \nu, \eta) \mathcal{E}_{\mathbf{b}}(k, t, \nu, \eta)\right]^{1 / 2}$ and $\left|\mathcal{H}_{M}(k, t, \nu, \eta)\right| \leqslant \mathcal{E}_{\mathbf{b}}(k, t, \nu, \eta) / k$, where $\mathcal{H}_{C}(k, t, \nu, \eta)$ and $\mathcal{H}_{M}(k, t, \nu, \eta)$ are the cross- and magnetic-helicity densities defined as the energy densities [Eqs. (3)]. Hence, in the limits $2 \pi / L \rightarrow 0, K \rightarrow \infty$ (with variables other than $k$ and $t$ suppressed for notational convenience), the cross helicity (for $q=1)$ satisfies

$$
\left|H_{C}(t)\right| \leqslant \int_{0}^{\infty} d k\left|\mathcal{H}_{C}(k, t)\right| \leqslant \frac{1}{2 t} \int_{0}^{\infty} d y\left[\Phi_{\mathbf{v}}(y) \Phi_{\mathbf{b}}(y)\right]^{1 / 2}
$$

and the magnetic helicity (for $q=1$ ) satisfies

$$
\left|H_{M}(t)\right| \leqslant \int_{0}^{\infty} d k\left|\mathcal{H}_{M}(k, t)\right| \leqslant \frac{1}{2 \sqrt{t}} \int_{0}^{\infty} d y \frac{\Phi_{\mathbf{b}}(y)}{\sqrt{y}} .
$$

The results obtained above apply for times $t<t_{*}$, where $t_{*}$ is the crossover time at which $L_{\mathbf{a}}(t)$ becomes equal to the size of the system (or the linear size of the simulation box in a DNS). For $t>t_{*}$, finite-size effects, which might well be nonuniversal, modify the decay of $E_{\mathbf{a}}(t)$.

\section{NUMERICAL RESULTS}

We now report on the numerical studies we have carried out to check the results given above. In most of our runs, we concentrate on the region $0<t<t_{*}$. We use double-precision arithmetic, but have checked in representative cases that our results are unaffected if we use quadruple-precision arithmetic.

Shell models comprise a set of ordinary differential equations containing suitable nonlinear coupling terms that re- 

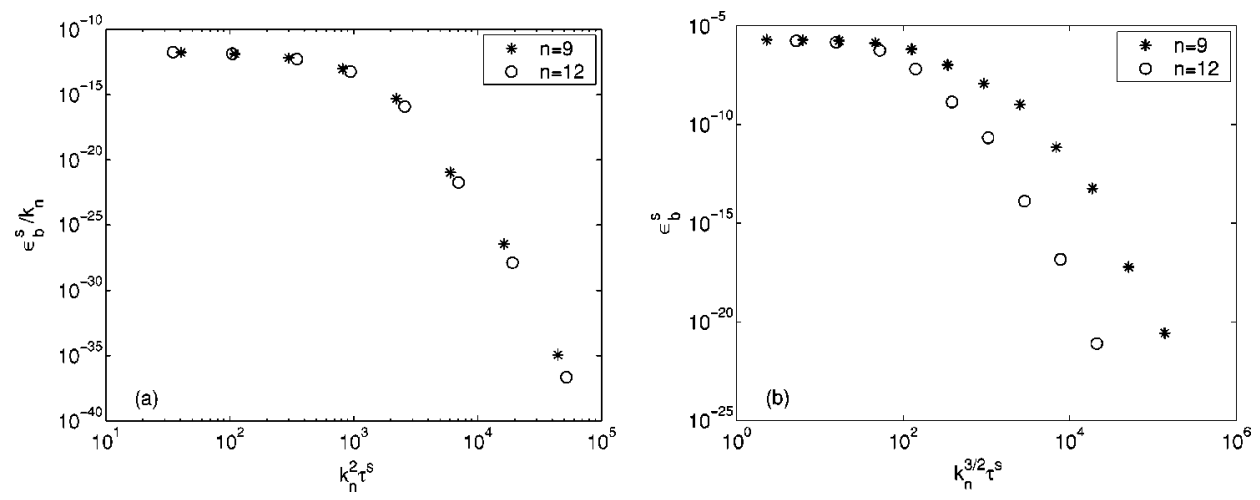

FIG. 1. (a) Plot of $\mathcal{E}_{b}^{s} / k_{n}$ vs $k_{n}^{2} \tau^{s}$ for shells $n=9$ and $n=12$ with $q=1$, illustrating the data collapse implied by the scaling form of Eq. (10). (b) The analog for $q=0$ illustrating the lack of data collapse.

spect the analogs of the conservation laws of the 3DMHD equations in the inviscid, unforced limit. They exhibit an energy cascade in the presence of viscosities. We confine our study to a shell model proposed in Refs. [11,12], which enforces all the ideal 3DMHD invariants in the inviscid, unforced case, reduces to the well-known Gledzer-OhkitaniYamada [14] model for fluid turbulence when magnetic terms are suppressed, has no adjustable parameters apart from the fluid and magnetic Reynolds numbers, and exhibits the same multiscaling (within error bars) as the 3DMHD equations.

The unforced shell-model equations are [15]

$$
\frac{d z_{n}^{ \pm}}{d t}=i c_{n}^{ \pm}-\nu_{+} k_{n}^{2} z_{n}^{ \pm}-\nu_{-} k_{n}^{2} z_{n}^{\mp},
$$

with the complex, scalar Elsässer variables $z_{n}^{ \pm} \equiv\left(v_{n} \pm b_{n}\right), i$ $=\sqrt{-1}$, and the discrete wave numbers $k_{n}=k_{0} 2^{n}\left(k_{0}\right.$ sets the scale for wave numbers), for shell index $n(n=1, \ldots, N$, for $N$ shells) with

$$
\begin{aligned}
c_{n}^{ \pm}= & {\left[a_{1} k_{n} z_{n+1}^{\mp} z_{n+2}^{ \pm}+a_{2} k_{n} z_{n+1}^{ \pm} z_{n+2}^{\mp}+a_{3} k_{n-1} z_{n-1}^{\mp} z_{n+1}^{ \pm}\right.} \\
& \left.+a_{4} k_{n-1} z_{n-1}^{ \pm} z_{n+1}^{\mp}+a_{5} k_{n-2} z_{n-1}^{ \pm} z_{n-2}^{\mp}+a_{6} k_{n-2} z_{n-1}^{\mp} z_{n-2}^{ \pm}\right]^{*} .
\end{aligned}
$$

Here $a_{1}=7 / 12, \quad a_{2}=5 / 12, \quad a_{3}=-1 / 12, \quad a_{4}=-5 / 12, \quad a_{5}$
$=-7 / 12, a_{6}=1 / 12$, and $\nu_{ \pm}=\nu \pm \eta$. Shell-model analogs of the total energy $\left[E_{T}^{s}=E_{v}^{s}+E_{b}^{s} \equiv(1 / 2) \Sigma_{n}\left(\left|v_{n}\right|^{2}+\left|b_{n}\right|^{2}\right)\right]$, the cross helicity $\left[H_{C}^{s} \equiv(1 / 2) \Sigma_{n}\left(v_{n} b_{n}^{*}+v_{n}^{*} b_{n}\right)\right]$, and the magnetic helicity $\left[H_{M}^{s} \equiv \Sigma_{n}(-1)^{n}\left|b_{n}\right|^{2} / k_{n}\right]$ are conserved if $\nu_{ \pm}$ $=0$. Here and in the following, the superscript $s$ stands for shell model. We solve Eqs. (15) numerically by an Adams-Bashforth scheme [16] (step size $\delta t^{s}=10^{-2}$ ) and use $N=22$ shells with $k_{0}=1 / 16$ and $\nu=\eta=10^{-4}$.

In decaying turbulence, it is convenient to measure time in units of the initial large eddy-turnover times. For our shell model these are $\tau_{a} \equiv 1 /\left(a_{r m s}^{0, s} k_{1}\right)$ with $a_{r m s}^{0, s} \equiv\left[\left\langle\Sigma_{n}\left|a_{n}^{0}\right|^{2}\right\rangle\right]^{1 / 2}$, the root-mean-square values of the initial velocities and magnetic fields. Since $\tau_{a}$ are calculated at the start of the simulation runs, when the velocity and magnetic fields differ only in phase (see below), here $\tau_{v}=\tau_{b}$ equal 18.7 for $q=1$ and 7.8 for $q=0$. Our runs are reported in terms of $\tau^{s} \equiv t / \tau_{a}$ ( $t$ is the product of the number of iterations and $\delta t^{s}$ ) and are ensemble averaged over 100 independent initial conditions with varying phases. We define $\operatorname{Re}_{v}^{0, s} \equiv v_{r m s}^{0, s} /\left(k_{1} \nu\right)$ and $\operatorname{Re}_{b}^{0, s}$ $\equiv b_{r m s}^{0, s} /\left(k_{1} \eta\right)$ to be the values of the initial fluid and magnetic Reynolds numbers (here $\operatorname{Re}_{v}^{0, s}$ and $\operatorname{Re}_{b}^{0, s}$ equal 34246 for $q$ $=1$ and 81920 for $q=0$ ). The initial velocity and magnetic fields are taken to be $v_{n}^{0}=k_{n}^{(1+q) / 2} e^{i \theta_{n}}$ and $b_{n}^{0}=k_{n}^{(1+q) / 2} e^{i \phi_{n}}$, with $\theta_{n}$ and $\phi_{n}$ being independent random variables distributed uniformly between 0 and $2 \pi$ (our shell-model energy
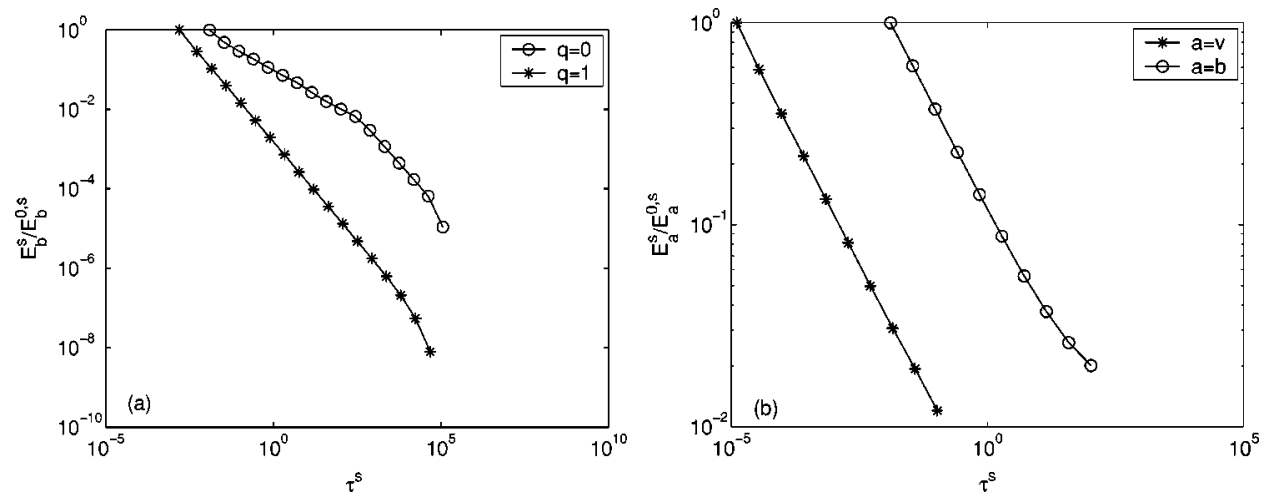

FIG. 2. (a) Log-log plots of the decay of the magnetic energy $E_{b}^{s}$ as a function of time $\tau^{s}$. The observed slope is $-1.000 \pm 0.001$ for $q$ $=1$ in agreement with Eq. (11). A nominal slope of $-0.66 \pm 0.01$ can be fit to the curve for $q=0$ in the range $\tau^{s}=1.28 \times 10^{-2}$ and $\tau^{s}=7.70$ $\times 10^{2}$. (b) Log-log plots of the decay of the kinetic and magnetic energies ( $E_{v}^{s}$ and $E_{b}^{s}$, respectively), without initial equipartition of energy $\left(E_{v}^{0, s}=10^{3} E_{b}^{0, s}\right)$ for $q=0$. 


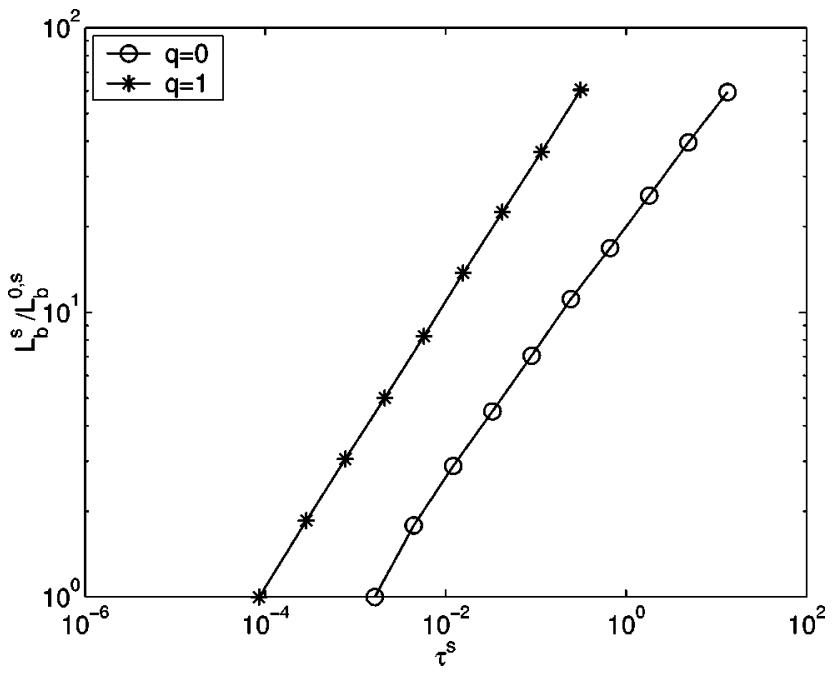

FIG. 3. Log-log plots of the growth of the magnetic integral scale $L_{b}^{s}$ (for $q=0$ and 1 ) as a function of time with $E_{v}^{0, s}=E_{b}^{0, s}$. The observed slope is $0.500 \pm 0.002$ for $q=1$ in agreement with the magnetic integral-scale analog of Eq. (12).

densities being defined as $\left.\mathcal{E}_{a}^{s} \equiv\left\langle\left|a_{n}\right|^{2} / k_{n}\right\rangle\right)$. In all our runs, $\left|H_{M}^{0, s}\right| / H_{M}^{\max , s} \lesssim 10^{-6}$, where $H_{M}^{\max , s} \simeq E_{T}^{0, s} / k_{1}$.

For $q=1$, Eq. (10) has the form $\mathcal{E}_{a}^{s}\left(k_{n}, \tau^{s}\right)$ $=k_{n} \Phi_{a}\left(k_{n}^{2} \tau^{s}, 1 / \nu^{2}, 1 / \eta^{2}\right)$. In Fig. 1(a) we show on a $\log -\log$ plot that a data collapse occurs for representative shells, here $n=9$ and $n=12$, when $\mathcal{E}_{b}^{s}\left(k_{n}, \tau^{s}\right) / k_{n}$ is plotted against $k_{n}^{2} \tau^{s}$. In Fig. 1(b) we show the analog for $q=0$ illustrating the lack of data collapse. Note, however, that the collapse improves as $k \rightarrow 0$; as we show below this leads to a power-law decay of $E_{b}^{s}$ over a limited range of $t$ and is related to the "permanence of large eddies."

In Fig. 2(a) we show the decay (for $q=0$ and 1) of the magnetic energy $E_{b}^{s}$ with an observed slope of $-1.000 \pm 0.001$ (with errors from least-square fits) for $q=1$. The final point on this graph for $q=1$ exhibits the beginnings of the crossover at $t=t_{*}$ where $L_{a}^{s}(t)$ becomes comparable to $k_{1}^{-1}$, the analog of the system size in the shell model.

For $q=0$, a nominal slope of $-0.66 \pm 0.01$ may be fitted over a portion of the curve between $\tau^{s}=1.28 \times 10^{-2}$ and $\tau^{s}$ $=7.70 \times 10^{2}$. This slope is in agreement with the law obtained from a hypothesis of permanence of large eddies, wherein the viscous terms are negligible at small $k$ in Eq. (10) leading to a decay law of -0.667 . The hypothesis of permanence of large eddies implies that if $E_{\mathbf{a}}^{0}(k) \sim k^{q}$, for $k$ $\rightarrow 0$, then the total energy decays as $E_{T} \sim\left(t-t_{0}\right)^{-2(1+q) /(3+q)}$ as discussed in the fluid turbulence context in Ref. [17].

So far we have used initial conditions with initial equipartition, i.e., $E_{v}^{0, s}=E_{b}^{0, s}$. We find that this is maintained during the decay, which is why we have shown plots of only the magnetic energy. For $q=1$ and $E_{v}^{0, s} \neq E_{b}^{0, s}$, both $E_{v}^{s}$ and $E_{b}^{s}$ decay as $t^{-1}$ for $0<t<t_{*}$ and maintain their initial ratio. In Fig. 2(b) we plot the temporal evolution of the kinetic and magnetic energies without initial equipartition, by setting $E_{v}^{0, s}=10^{3} E_{b}^{0, s}$ (here $\operatorname{Re}_{v}^{0, s}=10^{3} \operatorname{Re}_{b}^{0, s}$ ) for $q=0$. Unlike the case $q=1$, the kinetic and magnetic parts exchange energy here, but the dominant one (the kinetic energy in our plot) decays with a slope $-0.490 \pm 0.002$.

In Fig. 3, we plot (for $q=0$ and 1) the shell-model analog of the magnetic integral scale $L_{b}^{s} \equiv\left[\left\langle\left(\sum_{n}\left|b_{n}\right|^{2} / k_{n}\right) / \Sigma_{n}\left|b_{n}\right|^{2}\right\rangle\right]$ as a function of time. The observed slope is $0.500 \pm 0.002$ for $q=1$. In Figs. 4(a) and 4(b), we plot a temporal sequence (with separation in units of $\tau^{s}$ indicated in the legend) of the magnetic-energy density $\mathcal{E}_{b}^{s}$ as a function of wave number for $q=0$ and 1 . The kinetic-energy density evolves in a similar manner for the case of initial equipartition. Note that the evolution of these energy spectra illustrates quantitatively the qualitative notion of permanence of large eddies: As time progresses, the large- $k$ part of the spectra gets modified by viscous effects; however, the power-law part at small $k$ maintains its initial form for $t \lesssim t_{*}$. We have also explicitly checked that the bounds (13) and (14) are respected in our shell-model simulations.

For our DNS study of the 3DMHD equations, we use a pseudospectral method [18] to solve Eqs. (1) in a cubical box of side $2 \pi$ with periodic boundary conditions and $80^{3}$ Fourier modes. For the temporal evolution, we use an AdamsBashforth scheme (step size $\delta t=0.02$ ) and $\nu=\eta=10^{-2}$ (we exclude any hyperviscosities). We define the initial fluid and magnetic Reynolds numbers to be $\operatorname{Re}_{\mathbf{v}}^{0} \equiv 2 \pi v_{r m s}^{0} / \nu$ and $\operatorname{Re}_{\mathbf{b}}^{0}$ $\equiv 2 \pi b_{r m s}^{0} / \eta$ (here $\operatorname{Re}_{\mathbf{v}}^{0}$ and $\operatorname{Re}_{\mathbf{b}}^{0}$ equal 34 for $q=1$ and 17 for $q=0)$ with $a_{r m s}^{0} \equiv\left[\left\langle\Sigma_{\mathbf{k}}|\mathbf{a}(\mathbf{k})|^{2}\right\rangle\right]^{1 / 2}$ the root mean squares of the velocities and magnetic fields and initial large-eddy turnover times to be $\tau_{\mathrm{a}} \equiv 2 \pi / a_{r m s}^{0}$. As in the shell-model case, $\tau_{\mathbf{v}}=\tau_{\mathbf{b}}$ (here they equal 115.4 for $q=1$ and 228.2 for $q=0$ ) and $\tau \equiv t / \tau_{\mathrm{a}}$ ( $t$ is the product of the number of iterations and

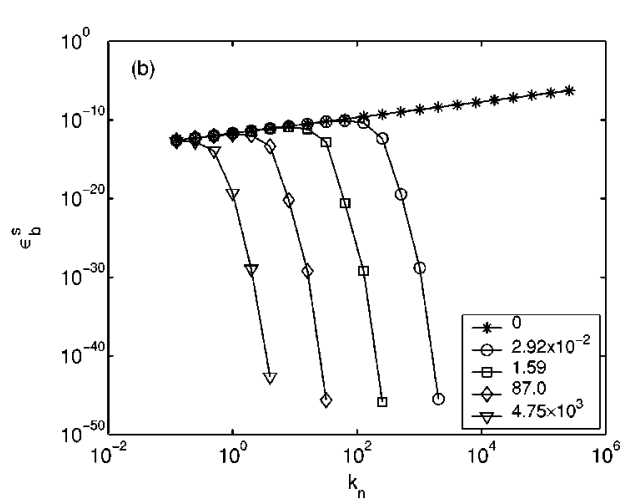

FIG. 4. Log-log plots of the MHD shell-model magnetic-energy density $\mathcal{E}_{b}^{s}$ as a function of wave number (temporal sequence with $\tau^{s}$ values indicated in the legend) for (a) $q=0$ and (b) $q=1$. 


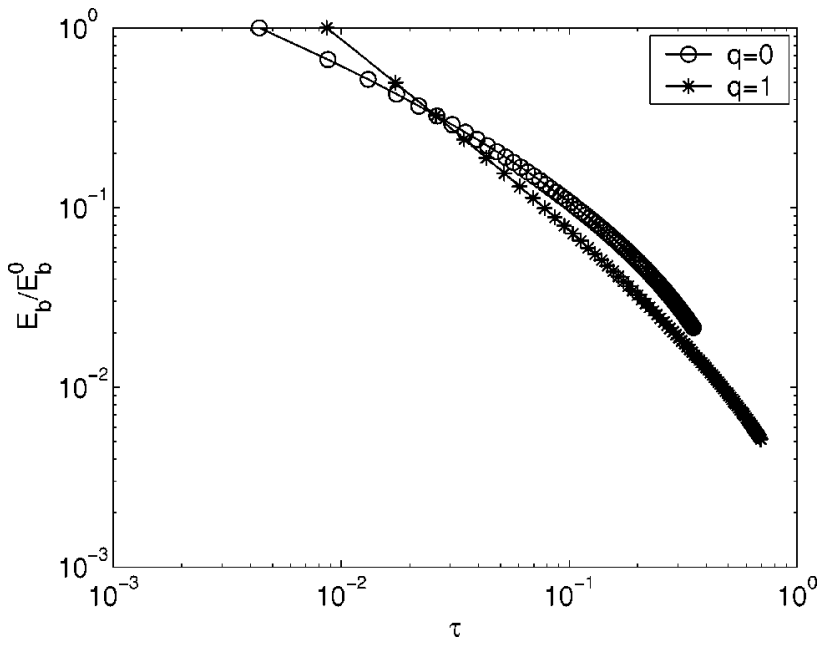

FIG. 5. Log-log plots of the magnetic energy $E_{\mathbf{b}}$ as a function of time for $q=0$ and 1 for 3DMHD from our $80^{3}$ DNS.

$\delta t)$. The initial velocity and magnetic fields are taken to be $\mathbf{v}(\mathbf{k}) \sim k^{q / 2} e^{i \theta_{\mathbf{k}}}$ and $\mathbf{b}(\mathbf{k}) \sim k^{q / 2} e^{i \phi_{\mathbf{k}}}$ with $\theta_{\mathbf{k}}$ and $\phi_{\mathbf{k}}$ being random variables distributed uniformly between 0 and $2 \pi$. Our DNS results are restricted to initial equipartition of energy between the velocity and the magnetic field.

In Fig 5 we plot the magnetic energy $E_{\mathbf{b}}$ as a function of time for $q=0$ and 1 . A slope of $-1.057 \pm 0.004$ is observed for $q=1$ between $\tau=8.7 \times 10^{-3}$ and $\tau=8.7 \times 10^{-2}$ in a reasonable agreement with Eq. (11). In Fig. 6 we plot the magnetic integral scale $L_{\mathbf{b}}$ as a function of time for $q=0$ and 1 . A slope of $0.401 \pm 0.003$ is observed for $q=1$ between $\tau=8.7 \times 10^{-3}$ and $\tau=8.7 \times 10^{-2}$. We believe that the slight discrepancy with the magnetic integral-scale analog of Eq. (12) arises because of the limited spatial resolution of the DNS. In Figs. 7(a) and 7(b), we plot a temporal sequence [in steps of $0.02 \tau(q=0)$ and $0.05 \tau(q=1)]$ of the magnetic-energy density $\mathcal{E}_{\mathbf{b}}$ as a function of wave number $k$ for $q=0$ and 1 . The small- $k$ part of the energy spectra in Figs. 7(a) and 7(b) changes somewhat more than their shell-model counterparts in Fig. 4. We believe this is because of the low spatial resolution of our DNS for 3DMHD. Our DNS results are presented here to complement our shell-model results and to show, in particular, that our general conclusions are not shellmodel artifacts.

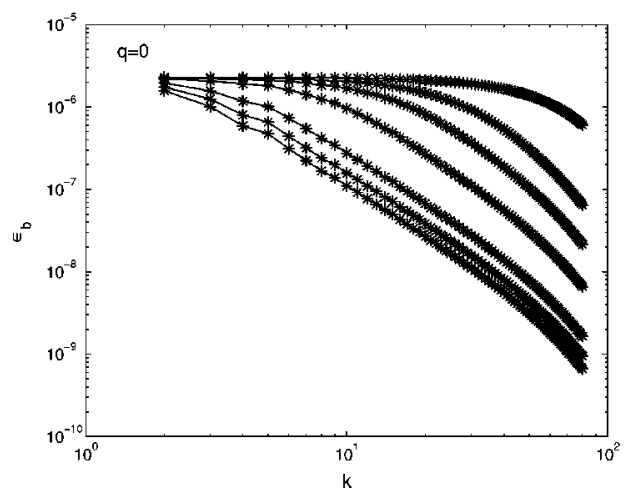

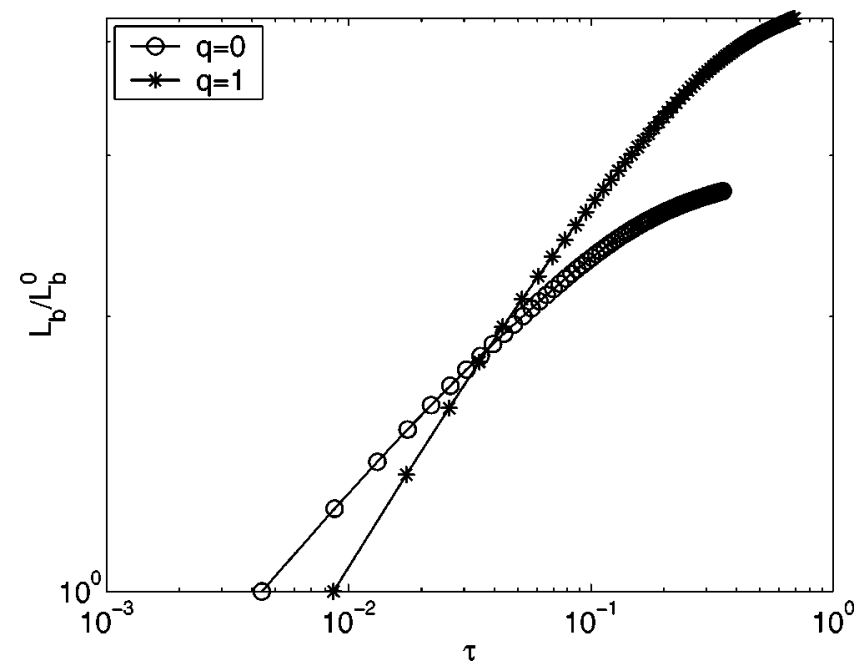

FIG. 6. Log-log plots of the magnetic integral scale $L_{\mathbf{b}}$ as a function of time for $q=0$ and 1 for 3DMHD from our $80^{3}$ DNS.

\section{DISCUSSION AND CONCLUSION}

To summarize, we have derived relations for the decay of the kinetic and magnetic energy, the growth of the Taylor and integral scales, and bounds for the cross and magnetic helicity in unforced, incompressible, homogeneous, and isotropic 3DMHD turbulence. We have confirmed our results numerically via shell-model and DNS studies. We show explicitly that our results about the power-law decay of the energies hold for times $t<t_{*}$, where $t_{*}$ is the time at which the integral scales become comparable to the system size. For $t<t_{*}$, our numerical results are consistent with those predicted by the principle of permanence of large eddies. Our study also has some implications for suggestions of "strong universality" in decaying turbulence as we discuss below.

In an early study [19], an expression for the kineticenergy density was hypothesized by assuming the existence of an "eddy viscosity." An energy decay similar to what we get for $q=1$ was then considered in the context of decaying fluid turbulence. However, this study did not consider the initial-condition dependence of the decay laws or the effect of the growth of the relevant length scales.

In a recent study [20], arguments have been given for strong universality in forced and decaying fluid turbulence in

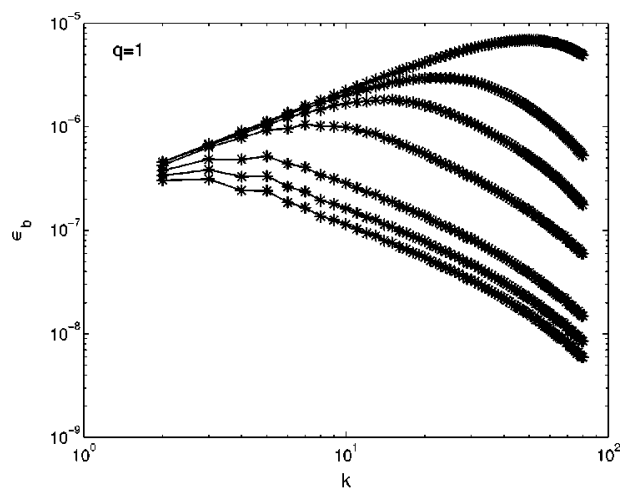

FIG. 7. Log-log plots of the magnetic-energy density $\mathcal{E}_{\mathbf{b}}$ as a function of wave number $k$ (temporal sequence in steps of $0.02 \tau$ for $q$ $=0$ and $0.05 \tau$ for $q=1$ ) for 3DMHD from our $80^{3} \mathrm{DNS}$. 
a shell model. For decaying turbulence, this means that scaling exponents of the $n$ th-order velocity structure functions and their coefficients in the isotropic sector, normalized by the mean energy flux, are universal and the same as those for structure functions that are obtained for forced turbulence. For the purpose of our discussion here, this would imply that, irrespective of the initial condition used, after an initial period of decay, the energy spectrum should evolve towards one with a part that goes as $k^{-5 / 3}$ (aside from multiscaling corrections which we are not concerned with here). We have been able to get results similar to those of Ref. [20] by using the MHD shell model [Eqs. (15)] and initial conditions as in Ref. [20]. In particular, if we start with equal initial kinetic and magnetic energies in the first two shells, the MHD-shellmodel simulations exhibit an energy cascade to higher shells. Once this cascade process is complete, the energy densities display a part that can be fitted to a $k^{-5 / 3}$ form and the integral scale moves towards small wave numbers. The point we would like to highlight here is that the evolution to a $k^{-5 / 3}$-type energy spectrum does not occur for the power-law initial conditions we have concentrated on in this paper. This is especially clear in the MHD-shell-model studies shown in Fig. 4. The reason for this is the permanence of large eddies: For small $k$, the energy spectrum retains the power-law dependence of the initial energy spectrum. Deviations from this initial power law become significant only for times $t>t_{*}$, at which the time-dependent integral scale $L_{\mathbf{a}}(t)$ becomes comparable to the size of the system. Given the parameters, such as $\nu$ and $\eta$, that we have used, by time $t_{*}$, the Reynolds number is sufficiently low that a fresh energy cascade to large $k$ is not established again. Thus, suggestions of strong universality should be made with caution, for they apply only to a class of initial conditions that does not include the power-law initial conditions used here for the decay of 3DMHD turbulence.

The initial conditions used in Ref. [9] are also qualitatively different from those that we use here in so far as they begin with a large energy in a few Fourier modes. Thus they develop energy cascades and do not explore the types of decay that are associated with the permanence of large eddies we concentrate on here.

\section{ACKNOWLEDGMENTS}

C.K. thanks Tejas Kalelkar for useful discussions, CSIR (India) for financial support, SERC (IISc) for computational resources, and R.P. acknowledges the Indo-French Centre for Promotion of Advanced Scientific Research (IFCPAR Project No. 2402-2) for support. We thank A. Basu and D. Mitra for discussions.
[1] G. Taylor, Proc. R. Soc. London, Ser. A 151, 421 (1935); A. Kolmogorov, Dokl. Akad. Nauk SSSR 31, 538 (1941); Proc. R. Soc. London, Ser. A 434, 15 (1991); P. Saffman, Phys. Fluids 10, 1349 (1967).

[2] A. Monin and A. Yaglom, Statistical Fluid Mechanics, edited by J. Lumley (MIT Press, Cambridge, 1975), Vol. 2.

[3] G. Comte-Bellot and S. Corrsin, J. Fluid Mech. 25, 657 (1966).

[4] M. Smith, R. Donnelly, N. Goldenfeld, and W. Vinen, Phys. Rev. Lett. 71, 16 (1993).

[5] M. Uberoi and S. Wallis, Phys. Fluids 10, 6 (1967); M. Mohamed and J. LaRue, J. Fluid Mech. 219, 195 (1990).

[6] W. George, Phys. Fluids A 4, 7 (1992).

[7] P. Ditlevsen, M. Jensen, and P. Olesen, e-print nlin.CD/ 0205055.

[8] P. Olesen, Phys. Lett. B 398, 321 (1997).

[9] D. Biskamp and W. Müller, Phys. Rev. Lett. 83, 2195 (1999); M. Christensson, M. Hindmarsh, and A. Brandenburg, Phys. Rev. E 64, 056405 (2001).

[10] We restrict ourselves to statistical invariance under rotations but not necessarily under reflections.

[11] A. Brandenburg, K. Enqvist, and P. Olesen, Phys. Rev. D 54, 1291 (1996); P. Frick and D. Sokoloff, Phys. Rev. E 57, 4155
(1998).

[12] A. Basu, A. Sain, S. Dhar, and R. Pandit, Phys. Rev. Lett. 81, 2687 (1998).

[13] The energy densities decay exponentially at large wave numbers in the far-dissipation range. We have therefore assumed convergence of the integrals appearing in Eqs. (11)-(14), which appears reasonable considering the presence of a naturally occurring viscous cutoff at large wave numbers.

[14] E. Gledzer, Sov. Phys. Dokl. 18, 216 (1973); K. Ohkitani and M. Yamada, Prog. Theor. Phys. 81, 329 (1989); L. Kadanoff, D. Lohse, J. Wang, and R. Benzi, Phys. Fluids 7, 517 (1995).

[15] There is a typographical error in the equations given in Ref. [12]. This does not affect their results. We have corrected this error in Eq. (15).

[16] D. Pisarenko, L. Biferale, D. Courvoisier, U. Frisch, and M. Vergassola, Phys. Fluids A 5, 10 (1993).

[17] U. Frisch, Turbulence: The Legacy of A. N. Kolmogorov (Cambridge University Press, Cambridge, 1996).

[18] S. Dhar, A. Sain, and R. Pandit, Phys. Rev. Lett. 78, 2964 (1997).

[19] W. Heisenberg, Proc. R. Soc. London, Ser. A 195, 402 (1948).

[20] V. L'vov, R. Pasmanter, A. Pomyalov, and I. Proccacia, Phys. Rev. E 67, 066310 (2003). 